\title{
Calibrando los lentes teóricos. Operacionalización y estrategias metodológicas para el análisis de las “identidades nacional populares"
}

\author{
Mauricio Schuttenberg \\ Universidad Nacional de La Plata. La Plata, Argentina. \\ Email: mauricioschuttenberg@gmail.com.
}

\begin{abstract}
Resumen: Este artículo apunta a reconstruir los pasos dados en la conformación del marco teórico de una tesis doctoral en el campo de las organizaciones populares en la historia reciente de la Argentina. En ese marco, se presenta la operacionalización de categorías teóricas con las cuales se abordó la dinámica política de dichas organizaciones durante el período 2003-2009 y se problematiza la estrategia metodológica. En resumen se propone reconstruir el proceso de construcción teórica de una investigación y los diferentes pasos que se siguieron desde la construcción del objeto, la discusión teórica y el aporte del estudio al concepto de identidad.
\end{abstract}

Palabras clave: Marco teórico, operacionalización, tesis, identidad, estrategia metodológica.

\section{Calibrating the theoretical lenses. Operationalization and methodological strategies for the analysis of the "popular national identities"}

\begin{abstract}
This article aims to reconstruct the steps in shaping the framework of a doctoral thesis in the field of popular organizations in the recent past in Argentina. In this context, we present the operationalization of theoretical categories with which they addressed the political dynamics of these organizations during the period 2003-2009 and will question the methodological strategy. In summary, it is proposed to reconstruct the theoretical construction of an investigation and the different steps followed from the construction of the object, the theoretical discussion and the research's contribution to the concept of identity.

Key words: Theoretical framework, operationalization, thesis, identity, methodological strategy.
\end{abstract}

\section{Calibrar as lentes teóricas. Operacionalização e estratégias metodológicas para a análise das "identidades nacionais populares"}

Resumo: O presente trabalho tem como objetivo reconstruir os passos da formação do quadro de uma tese de doutorado no campo das organizações popula- 
res na história recente da Argentina. Neste contexto, apresentamos a operacionalização das categorias teóricas com que são abordadas a dinâmica política dessas organizações durante o período 2003-2009 e vai questionar-se a estratégia metodológica. Em resumo pretende-se reconstruir o processo de construção da investigação teórica e os diferentes passos seguidos a partir da construção do objecto, a discussão teórica e a contribuição do estudo para o conceito de identidade.

Palavras-chave: enquadramento teórico, operacionalização, teses, identidade, estratégia metodológica.

\section{Introducción}

En este artículo apuntamos a reconstruir los pasos dados en la conformación del marco teórico de una tesis doctoral ${ }^{1}$ en el campo de las organizaciones populares en la historia reciente de la Argentina. Con este objetivo se desarrollarán las distintas etapas por las que atravesó la investigación hasta presentar algunas reflexiones acerca de los alcances y limitaciones que la perspectiva teórica tomada tiene a la hora de abordar procesos políticos contemporáneos.

Lo que el trabajo pretende es problematizar la compleja operacionalización conceptual de una categoría tan vasta como la de identidad. En este sentido, en los últimos años el concepto de identidad aparece como referencia de una gran cantidad de estudios sociales de temáticas diferentes. Asimismo, no sólo los diversos campos donde se aplica el concepto, sino más bien la polisemia con la cual se lo utiliza se presenta como un problema a tener en cuenta para el análisis político.

El artículo se propone entonces desarrollar el proceso de construcción del marco teórico para el análisis de las identidades políticas de las organizaciones "nacional populares"2 en su complejidad que permita la reflexión en torno a los actos de identificación, las decisiones, los imaginarios sociales y las reconfiguraciones identitarias de tales organizaciones. Tal decisión se sostiene en que este entramado teórico nos permitirá entender la acción política, en la medida en que es la identidad la que posibilita a los actores significar las situaciones y escoger en consecuencia ciertas alternativas de acción. En segundo término, se presenta la operacionalización de categorías teóricas con las cuales se abordó la dinámica política de dichas organizaciones durante el período 2003-2009.

\section{Primeros pasos: el estado del arte}

La primera etapa consistió en la investigación bibliográfica, que puede definirse como aquella fase de la investigación donde se explora qué se ha escrito en la comunidad científica sobre un tema o problema determinado. Como señala Curzel (2005) permite apoyar la investigación 
que se desea realizar, conocer experiencias realizadas, continuar investigaciones, plantear diferentes aspectos de una línea de trabajo, buscar información actualizada, seleccionar un marco teórico, etc.

Teniendo en cuenta lo anterior la primer tarea a la hora de estructurar la investigación de tesis doctoral fue la recopilación y problematización de lo trabajado en el campo académico donde se inscribía la tesis, es decir los estudios sobre organizaciones populares y movimientos sociales en la Argentina contemporánea con énfasis en la dinámica de las organizaciones "nacional populares". En ese marco, se destacaba que las investigaciones sobre el movimiento "piquetero"3 partieron en general de analizar sus formatos organizativos, sus formas de protesta y en algunos casos su identidad social en tanto movimiento de trabajadores de desocupados. Asimismo, la mayoría de los trabajos que abordan la etapa posterior a 2003 dan cuenta del paso del ciclo de protesta a una mutación de la misma a partir de la asunción de Kirchner, poniendo el acento en cómo desde el Estado se condicionó la acción política de las organizaciones. No se indagó en la misma medida lo que ocurrió “abajo", es decir, cómo se vivenció en las organizaciones la etapa que se abrió en 2003 y cómo algunas de ellas construyeron nuevos posicionamientos y rearticularon en el camino nuevos y viejos componentes identitarios.

El cambio del escenario político a partir de 2003 generó en efecto una importante modificación en el modo de vinculación entre Estado y organizaciones de desocupados. La estrategia que desarrolló el gobierno de Kirchner fue incorporar a su proyecto político a algunas de esas organizaciones, lo que a su vez potenció, reactualizó y resignificó la posibilidad de que ellas se articulen en torno a una identidad "nacional-popular". Ahora bien, la inserción de algunos grupos en el espacio kirchnerista tendió a explicarse en un principio, en numerosos trabajos del período (Campione y Rajland, 2006; Borón, 2007; Battistini, 2007, Svampa, 2006), en términos de cooptación concepto que desde mi perspectiva resulta insuficiente para dar cuenta de los posicionamientos de un número importante de organizaciones en tanto subyace en él un argumento negador de la producción política de estos actores.

Si bien esta idea es el denominador común del proceso político abierto en 2003, aparece formulada haciendo hincapié en distintas causas. Se la caracterizará como estrategia estatal de contención de la protesta, como reemplazo al nivel colectivo de la matriz clientelar, como manifestación de la debilidad de los sectores y organizaciones populares y como capacidad de "volver al orden" del Partido Justicialista ${ }^{4}$.

Desde esta perspectiva teórica, la idea de cooptación explica entonces el posicionamiento y el reordenamiento político posterior a 2003. No obstante, en los últimos años salieron a la luz algunos trabajos que comenzaron a cuestionar las hipótesis de cooptación y empezaron a problematizar la dinámica de los movimientos sociales en la presidencia de Kirchner. Esta relación comienza a ser interpretada como una decisión conciente de las 
organizaciones ante el desafío de reposicionarse frente a un contexto de reflujo de la movilización y, fundamentalmente a redefinir sus estrategias políticas frente a un gobierno que construyó rápidamente su legitimidad de ejercicio apelando a la oposición al modelo neoliberal a través de un imaginario productivista y distributivo que recuperaba buena parte de las demandas que habían permitido la articulación de la protesta (Pérez, 2008) Massetti (2009) y Gómez (2009).

En este marco, el objetivo de la investigación fue recuperar la historia de esas organizaciones y comprender que la dinámica política que comienza en 2003 no es necesariamente para ellas una ruptura, cooptación y abandono de prédicas revolucionarias, sino que se trata de un proceso de construcción y reconstrucción de las identidades "nacional populares", en donde se pone en juego la historicidad sedimentada de las organizaciones en distintos contextos. Esto posibilita desarrollar una lectura de largo plazo de las mutaciones de las identidades de las organizaciones para captar los elementos de continuidad y los antecedentes precursores que se corresponden con desarrollos y posicionamientos ulteriores.

De esta forma el problema de investigación buscaba dar respuesta a ¿Qué espacios políticos se abrieron a partir de 2003 para algunas organizaciones populares? ¿En qué se sustentan las nuevas interpretaciones que las organizaciones realizan de las nuevas relaciones con el Estado? ¿Quién es el "otro" que se construye como enemigo y que articula las solidaridades internas? ¿Cómo se resignifica el pasado y las tradiciones políticas en las lecturas del presente? ¿Desde qué lugares se reivindica la identidad peronista? ¿Sobre qué figuras y cómo se construyen estos relatos? ¿Cómo son las instancias de producción de identidad? ¿Cuáles fueron los quiebres en esas producciones y qué hechos las marcaron? ¿Cómo es la dinámica específica de cada una de las organizaciones en el período 2003-2009? Para contestar estas preguntas era necesario construir un "lente teórico" sensible a los procesos de reconstrucción identitaria de esas organizaciones que, surgidas en la lucha contra el neoliberalismo, construyen sus vínculos con el gobierno de Kirchner.

\section{La problematización del marco teórico}

El análisis de los movimientos sociales para las ciencias sociales tuvo su auge cuando muchas de las certezas acerca de las identidades colectivas homogéneas se fueron desmoronando (Schuster, 2005). A partir de los años 60 se da un cambio en la unidad de análisis de los estudios sociológicos. El movimiento obrero deja su lugar en las indagaciones al surgimiento de lo que se denominó "nuevos movimientos sociales"5. De la mano de lo anterior se rompe con las clasificaciones y análisis que partían del concepto de clase social. En "las nuevas sociedades” reflexivas (Beck, 2002 y 2004), cambiantes el conflicto parece diseminarse por todo el espacio social y no será ya, sólo, el movimiento obrero el sujeto político que encarne el conflicto societal. 
En ese contexto cobraron forma dos vertientes fundamentales, una en Estados Unidos -conocida en principio como "teoría de la movilización de recursos”- y la otra en Europa -o teoría de los nuevos movimientos sociales propiamente dicha, cada una proponiendo contribuciones particulares al análisis de los movimientos ${ }^{6}$. Si en las propuestas norteamericanas ${ }^{7}$ era el carácter estratégico de la acción colectiva lo que proporcionaba el principal nexo de unión, formular los nuevos movimientos sociales como la reacción a los cambios estructurales de las sociedades avanzadas será el elemento unificador de los teóricos europeos. Las diferencias entre las dos escuelas de pensamiento obedecían a las tradiciones intelectuales dominantes en cada cultura: individualista en Estados Unidos y estructuralista en Europa (Tarrow, 1997 y 1999).

En la vertiente norteamericana, las investigaciones sobre los movimientos sociales se centraron en sus formas de acción política y sus posicionamientos estratégicos que fueron analizados desde el concepto de acción colectiva. Esta noción apuntaba a dar cuenta de las motivaciones por las cuales se producía efectivamente la movilización de colectivos. En este marco, en un primer momento la cuestión de la identidad de los movimientos sociales quedó relegada en la búsqueda de elementos "externos" que pudieran explicar la movilización y la acción política. La acción entonces se explicó a partir de las oportunidades políticas y la aparición coyuntural en el sistema político de elementos que promueven a la participación.

Allí encontraremos entonces algunos elementos que irán desarrollándose ligados a la cuestión de las identidades pero atravesados por una constante de la escuela norteamericana que es la de estrategia ${ }^{8}$. Todas las construcciones simbólicas, "las banderas", de las organizaciones fueron pensadas en términos de construcciones con fines específicos, o elementos a los que, mediante una operación estratégica, los líderes tomaban con un fin determinado.

Por otro lado, el problema de la identidad fue abordado como el eje central por la denominada teoría de los movimientos sociales o vertiente "europea”. Se concibe como una contrapropuesta para comprender las luchas y reivindicaciones sociales a partir del rescate del actor y del sujeto. La perspectiva de los nuevos movimientos sociales que se origina en Europa trata de poner en evidencia las dimensiones culturales y sociales de las prácticas colectivas. Para esta línea los actores sociales, por medio de sus prácticas colectivas, reinterpretan normas y valores, creando nuevos significados para los estrechos límites de la acción política. Esta línea de investigaciones compartía un desencanto con el marxismo estructural que privilegiaba el análisis de las contradicciones de las clases definidas desde la economía. También les interesaba comprender las movilizaciones que se desarrollaban en sus países a fines de los años sesenta, las cuales aparecieron como nuevas y distintas de las anteriores analizadas por la izquierda política.

No obstante, a la hora de pensar un andamiaje teórico para el análi- 
sis de las organizaciones populares en la Argentina contemporánea, los conceptos desarrollados anteriormente de las teorías del enfoque político y de la movilización de recursos, resultan poco sensibles para el análisis de los procesos constituyentes y de las reconfiguraciones identitarias de las organizaciones populares por prestar escasa atención a la dimensión simbólica de las mismas, subsumiendo esa producción detrás de una concepción más estratégica de la política.

Asimismo, los enfoques identitarios como los de Melucci, Pizzorno y Touraine, basados en el concepto de la identidad colectiva de los nuevos movimientos sociales necesitan una “adaptación” para el análisis de organizaciones que se caracterizan por la construcción de fuertes vínculos y tradiciones con formas de organización política que pueden ser vistas como una continuidad de reivindicaciones centradas en la defensa de los derechos de los trabajadores y no en demandas particulares como sería la característica de los movimientos sociales (Gurrera, 2005).

Por lo tanto, estas miradas requieren ser complementadas por otras perspectivas a la hora de pensar la identidad de las organizaciones de trabajadores desocupados y las organizaciones populares que de ellas se desprenden. En estos casos “lo político” aparece como central, tienen además proyectos ideológicos y no son fragmentarios. Asimismo, estas identidades no sólo no rechazan las totalizaciones sino que las reconstruyen. Por ende, se hace necesaria la conceptualización de una matriz analítica capaz de dar cuenta de la especificidad de las reconfiguraciones identitarias de las organizaciones populares ocurridas en los últimos años en la Argentina.

\section{La operacionalización del concepto de identidad política para el estudio de organizaciones populares}

La operacionalización de conceptos teóricos constituye una fase intermedia en el proceso de investigación. Implica que se ha definido un tema de estudio y antecede al diseño de indagación empírica, instrumento de recolección de datos La operacionalización se define como: “el proceso de llevar una variable de nivel abstracto a un plano operacional (...) lo que se traduce en el establecimiento de significados para los términos del estudio, y en la estipulación de operaciones o situaciones observables, en virtud de lo cual algo quedará ubicado en determinada categoría o será medido en cierto aspecto.” (Canales, Alvarado, y Pineda, 1989: 111 y 112). Resumiendo, la operacionalización implica la división del concepto en conceptos menores, en dimensiones que son subdivisiones semánticas del concepto. Son los aspectos discernibles, tienen la propiedad de ser parte de una totalidad mayor.

Abordar las organizaciones populares durante la etapa posneoliberal implica pensar los procesos de reconfiguración de las identidades políticas y exige “adaptar" ciertos conceptos a un campo de aplicación diferente al 
de movimiento social. En este sentido un primer paso fue especificar y diferenciar el concepto de organizaciones populares respecto al de movimientos sociales, para luego explorar las herramientas analíticas que nos permitan estudiar las identidades en su dimensión política. La definición de un movimiento social se basa en el proceso de constitución de una identidad colectiva, fuera del ámbito de la política institucional por el cual se dota de sentido a la acción individual y colectiva (Revilla Blanco, 1994). Schuster (2005) define a un movimiento social a partir de una serie de características tales como tener una identidad común, que dicha identidad tenga continuidad en el tiempo, que exista una expansión en el espacio del sistema identitario de acciones y que la línea de identidad de las acciones pueda reconstruirse a través de sucesivas emergencias y ocultamientos.

El concepto de movimiento social alude fundamentalmente a tres características que son: la identidad en común a través del tiempo, la construcción de demandas que exceden las reivindicaciones de clase y una estructuración no jerárquica. En este trabajo nos referiremos a organización en lugar de movimiento social puesto que si bien comparte un aspecto de la definición del mismo, es decir, la existencia de una sólida identidad colectiva, la cuestión del horizontalismo y el carácter "estructurado" de las mismas son elementos que las alejan de esa concepción.

La noción de organizaciones populares hace referencia al espacio de conformación de estas en el intersticio que dejaron los partidos políticos y los sindicatos (Masetti, 2009) y en su origen se encuentran dos procesos: el desarrollo de un nuevo paradigma de acumulación política surgido a nivel local y las experiencias colectivas de resolución de necesidades a partir de las crisis económicas de los años 80 y 90 . El concepto de "organizaciones populares" se ubica en una dimensión analítica diferente a la de movimiento social en tanto hace alusión a un referente empírico concreto más que a lógicas de acción. A diferencia de los movimientos sociales, las organizaciones se caracterizan por tener un relativo grado de estructuración organizativa que retoma antiguas tradiciones e identidades políticas, por articular demandas de carácter económico y por posicionarse y trazar estrategias en el ámbito político institucional.

Partir entonces de la pregunta acerca de la reconfiguración de las identidades políticas conlleva a establecer algunas definiciones previas de lo que ello implica ${ }^{9}$. El concepto de identidad aparece como referencia de una gran cantidad de estudios sociales de temáticas diferentes. Diversos autores (Arfuch, 2002; Brubaker y Cooper, 2001; Dubet, 1989) resaltan que las ciencias sociales se han rendido ante la palabra identidad. Identidad tiende a significar demasiado o muy poco según sea el enfoque. Según estos autores, en los últimos años tendió a significar poco puesto a que la prevaleciente postura constructivista frente a la identidad -en un intento de suavizar el término para liberarlo de la carga de esencialismo mediante la estipulación de que las identidades son construidas, fluidas y múltiplesdejó sin argumento para hablar sobre identidades ${ }^{10}$. Estas argumentaciones 
reclaman una precisión conceptual de lo que implica pensar la dinámica política a partir de la noción de identidad.

Debido a que el término “identidad” parece semánticamente inseparable de la idea de "permanencia", es necesario pensar un concepto capaz de captar tanto "lo permanente” (sedimentado) de una identidad política como su continua recreación, es decir sus dimensiones estáticas y dinámicas. Buscamos reconstruir las mutaciones y a su vez lo sedimentado que estructura una identidad política. En este trabajo apuntamos a pensar el campo "nacional-popular", no como una identidad esencial sino como una construcción histórica en donde las diversas identidades ponen en juego procesos de construcción que incluyen elementos sedimentados a partir de sus experiencias políticas previas y elementos que se activarán al calor de la coyuntura y las alternativas del proceso político.

Para analizar las formas de constitución y reconfiguración de las identidades políticas "nacional populares”, se tomaron algunos conceptos claves de Laclau como: hegemonía, antagonismo, puntos nodales, significantes vacíos, significantes flotantes, lógica de equivalencia, lógica de la diferencia. La importancia del análisis del discurso parte de un principio ontológico que es comprender a lo social como un espacio discursivo, con lo cual la concepción de estructuración de lo social responde a un modelo retórico. La noción de discurso de Laclau refiere a toda relación de significación. Desde esta perspectiva, el discurso no sería producido por un sujeto que fuera su agente, sino a la inversa, el sujeto social sería una realización del discurso (Biglieri, 2007).

La identidad implica entonces un proceso dinámico de construcción de significados. En este punto recuperamos el concepto de configuración identitaria propuesto por De la Garza (1997, 2001). Entender la identidad como una configuración permite concebirla como un proceso móvil que articula elementos heterogéneos que tendrán distintos lugares en esa cadena significante en las distintas coyunturas.

Estos procesos constituyentes permiten pensar que la reconstrucción de las identidades y la atribución de sentidos por parte de las mismas, se establecen como elementos centrales para el análisis de los posicionamientos políticos. Es necesario producir un conocimiento sobre las formas en que los significados son construidos y sobre cómo los diferentes procesos políticos son significados en las diversas coyunturas.

Los elementos constitutivos de la identidad se articulan, en una dinámica siempre inacabada y abierta (Zemelman, 1995 y 1997). Esto implica la necesidad de analizar una dimensión autónoma de los aspectos estructurales que inciden en los mecanismos de identificación y en la conformación de configuraciones identitarias.

Pensar las identidades en estos términos abre la posibilidad de entender la conformación de nuevas configuraciones. No obstante, cada 
reordenamiento, cada incorporación, cada modificación, cada reconfiguración, genera reacomodamientos donde se pueden identificar continuidades y cambios (Barros, 2006a y2006b). Dentro de esos elementos que se reconfiguran tiene importancia el análisis de los puntos nodales donde se condensan los significados. En efecto, en la configuración identitaria no todos los significados tienen el mismo peso para la articulación, algunos códigos pueden adquirir primacía y opacar a otros que permanecen subalternizados, pero que pueden emerger y conformarse en "articulantes" de la red de códigos y por lo tanto también del proceso colectivo de dar sentido (Retamozo, 2009a y 2009b). La identidad aparece entonces como el producto de sucesivas identificaciones y se constituye entonces en la tentativa de dominar y detener el flujo de las diferencias para construir un discurso. En ese marco, los puntos nodales condensan sentidos y fijan parcialmente las identidades. Estos puntos nodales constituyen de alguna forma significantes centrales que estructuran una cadena significante y estabilizan temporariamente una identidad (Laclau, 2006).

A la hora de operacionalizar este marco teórico al plano empírico, se planteó reconstruir los puntos nodales sobre los cuales las organizaciones bajo estudio construyeron su identidad y cómo estos fueron mutando en las diferentes etapas. La forma de buscar una referencia empírica se basó en la búsqueda de las concepciones que las organizaciones fueron desarrollando sobre el Estado, la democracia, sobre el sujeto histórico del cambio social, sobre cómo debía darse esa transformación social, sobre las interpretaciones de la historia, la construcción de antagonismos y sobre los diversos momentos y figuras históricas con las cuales las organizaciones se identificaron.

Como bien explica Sautú (2003) la estructura básica de una investigación se comprende por la formulación de un objetivo a partir de una perspectiva teórica específica, la producción de la prueba empírica realizada en forma sistemática y controlable y, por la inferencia de conclusiones. Sin embargo, esta estructura no implica momentos estancos y separados donde aparece por un lado lo "teórico" y por otro lo "metodológico". La estrategia metodológica debe encuadrarse en relación a los objetivos del investigador y al marco teórico propuesto.

En este sentido, las técnicas cualitativas fueron utilizadas para indagar sobre los procesos de conformación de las diversas identidades, las experiencias colectivas, los significados y representaciones que los sujetos tienen de su participación en la acción. En este plano, el recorte de las organizaciones a analizar -el Movimiento Evita ligado al peronismo de izquierda, el Movimiento Libres del Sur con raíces en la izquierda nacional y el Movimiento de Unidad Popular de tradición anarquista- apuntó a profundizar en el espacio político "nacional popular" y a rastrear la heterogeneidad de representaciones, imaginarios, identidades y posicionamientos políticos que se insertarán en el kirchnerismo. Para ello se optó por una selección múltiple intencional de $\operatorname{casos}^{11}$ basada en la diversidad, es decir, el trabajo apuntó a mostrar las distintas identidades y trayectorias que con- 
fluirán en el kirchnerismo. La decisión de trabajar con casos múltiples refuerza la posibilidad de contrastación de datos, comparación por semejanzas y por diferencias que pondrán de manifiesto las diversas formas que asumió el proceso político en las identidades políticas seleccionadas.

La investigación se centró en un análisis del discurso político de las organizaciones entendiendo por ello que como en todo comportamiento social, la acción política no es comprensible fuera del orden simbólico que la genera y del universo imaginario que ella misma engendra. Se planteó entonces reconstruir los puntos nodales sobre los cuales las organizaciones bajo estudio construyeron su identidad y cómo estos fueron mutando en las diferentes etapas.

Teniendo en cuenta estos objetivos se diseñó una estrategia metodológica cualitativa centrada en la búsqueda y análisis de documentos públicos y de circulación interna de las tres organizaciones. En tanto, también se trabajó con las publicaciones que las organizaciones tuvieron en las distintas etapas: Revista Evita del Movimiento Evita, Revista En Marcha de Patria Libre y Revista Patria Grande de Libres del Sur y con respecto al MUP se tomaron centralmente los documentos en los diversos períodos abordados.

A su vez se realizaron entrevistas semiestructuradas a dirigentes de las tres organizaciones desde agosto de 2007 a julio de 2009. Los cuestionarios apuntaron a recabar información sobre las distintas interpretaciones, posicionamientos que las organizaciones analizadas tuvieron desde su conformación hasta el presente y sobre las trayectorias y tradiciones de la militancia que las compone. Cabe destacar que para la reconstrucción histórica del proceso de formación y de las acciones de los años 90’ nos basamos en el análisis de documentos y publicaciones de las organizaciones. En este punto existían y existen diferencias entre las organizaciones. En algunos casos se pudo reconstruir las trayectorias y posicionamientos a partir de los documentos emitidos por las organizaciones en cada etapa y en otros, se debió apelar a las entrevistas a partir de la falta de documentos y fuentes escritas en el propio momento.

Además los procesos de conformación y las discusiones en cada coyuntura eran elementos que sólo podían recuperarse a través de la entrevista puesto que los documentos o publicaciones de prensa dan la "visión oficial" de cada una de las organizaciones y no permite visualizar las tomas de decisión y los elementos que allí se ponen en juego. Alonso (1998) señala que las entrevistas en profundidad posibilitan la comprensión "del texto" en su "contexto social” y en la historicidad de sus planteamientos, desde la reconstrucción de los intereses de los propios actores. De esta forma, se buscó recuperar el sentido de lo vivido por los sujetos, el significado que se les dieron a sus prácticas y acciones. Siguiendo este enfoque, el habla es reveladora de condiciones estructurales, de un sistema de valores, normas y símbolos y, al mismo tiempo, refleja las representaciones de grupos determinados en condiciones históricas, socio-económicas y culturales específicas. 
Las entrevistas y el análisis documental estuvieron apuntados a identificar los núcleos donde se sedimentan los sentidos de estas identidades. La descripción profunda de estos puntos nodales nos permitió acceder a los elementos acumulados por las experiencias políticas previas a 2003 y a cómo estas tomarán nuevas significaciones en el contexto del gobierno de Néstor Kirchner. Teniendo en cuenta los objetivos que nos habíamos propuesto, se diseñó una guía de entrevista que contenía las principales preguntas de investigación y que permitía la comparación entre miembros de distintas organizaciones para ver las similitudes y diferencias en torno a los puntos nodales del discurso de cada una de las identidades. A su vez para el análisis documental se construyeron una serie de categorías centradas también en los puntos nodales con el fin de agrupar los fragmentos del discurso. Esto permitió no sólo comparar sino observar la dinámica política de las organizaciones en torno a los principales núcleos ideológicos. Así se pudo dar cuenta de mutaciones en las formas de pensar el Estado, el peronismo y los caminos hacia la transformación social, cuestiones estas que explican los posicionamiento políticos. Esto último constituyó el eje de nuestra tesis puesto que se explica la acción política a partir de las dimensiones ideológicas que las organizaciones fueron construyendo durante el período analizado y se cuestionan las explicaciones centradas en que el comportamiento político obedece a cómo el Estado coopta estas organizaciones a partir de la manipulación de un discurso "nacional y popular” o de la participación en programas sociales a cambio de apoyo.

De esta forma, se apuntó a comprender cómo una nueva coyuntura sitúa a una organización en la necesidad de asumir su propia recreación. Esta no es sólo la manifestación de la existencia previa sino una nueva instancia de producción de identidad. La coyuntura de 2003 que inauguró una etapa en donde las organizaciones participarán del gobierno de Néstor Kirchner. Esa relación se forjó a partir de una compleja articulación política que denominamos "puentes discursivos".

Estos puentes expresan el pasaje de una determinada configuración identitaria a otra, explican la reconstrucción que cada identidad realiza de sí misma en cada coyuntura. Al comienzo del artículo nos habíamos preguntado por la posibilidad de construir un concepto de identidad capaz de captar tanto lo sedimentado como lo dinámico de una identidad. De esa forma, los puentes discursivos constituyen un concepto operacional que permite analizar las dos dimensiones de la dinámica identitaria.

Ahora bien, para analizar ese proceso dinámico es necesario retomar la distinción que Laclau (2005) realiza entre identidad y acto de identificación. La identidad aparece como el producto de sucesivas identificaciones imaginarias. El acto de identificación, por su parte, es la fundación de una nueva significación y, como tal, la posibilidad de desestabilización de toda identidad objetivada. El acto de identificación es la institución de nuevos sentidos más allá de la simple repetición, y que como tal puede materializarse en la aparición de una nueva nominación que articulará discursos dispersos atribuyéndolos a una nueva unidad de referencia. 
De esta manera, la identificación es contingente e instituye nuevos sentidos que se articulan con la identidad política que es definida entonces “como el conjunto de prácticas sedimentadas, configuradoras de sentido, que establecen, a través de un mismo proceso de diferenciación externa y homogeneización interna, solidaridades estables, capaces de definir, a través de unidades de nominación, orientaciones gregarias de la acción en relación a la definición de asuntos públicos” (Aboy Carlés, 2001: 54).

En este sentido, la identificación construye la identidad puesto que la entendemos en tanto articulación de significados para dar sentido a una situación. Esto supone concebir un espacio analítico en la articulación de significados que construyen a partir de la apelación a elementos del campo “nacional y popular” y cómo cada una de las organizaciones articulará los mismos en sus cadenas de significación. De este modo, las identidades serían producto de determinadas articulaciones subjetivas parcialmente estables que incorporan aspectos identitarios, dimensiones de reconocimiento, proyecto y acción. Interesa entonces profundizar en la relación entre lo dinámico y lo sedimentado (Retamozo, 2008).

Las identificaciones que una organización realiza se entrelazan con sus experiencias sedimentadas. Esta relación abre un espacio para el análisis empírico. La pregunta refiere entonces a cómo lo sedimentado o instituido se estructura y estructura a su vez a lo instituyente en los discursos de las organizaciones. Pensar la dinámica política en éstos términos nos abre la posibilidad de analizar cómo los diversos grupos van reestructurando sus identidades y sus posicionamientos y cómo la constitución de límites y antagonismos posibilita el realineamiento de fuerzas y la rearticulación en busca de un nuevo intento de "sutura” de las identidades. El presente marco teórico pretende rescatar los momentos de estabilización y desestabilización de las identidades a partir del análisis de los períodos en que se debilitan las articulaciones hegemónicas.

Ahora bien, esas reconfiguraciones identitarias son articulaciones de elementos significantes que en distintas coyunturas tendrán distintas posiciones, pero operan dentro de lo que podríamos denominar como una matriz ideológica que son las doctrinas previas que generan una determinada forma de identificación política, es decir un mínimo denominador común de la matriz ideológica a la que se ven obligadas a legitimar en tanto que base de validez que hace posible su propio discurso (Perelli, 1986).

\section{Conclusiones}

Este artículo apuntó a presentar los pasos fundamentales del proceso de construcción del marco teórico, su problematización y conceptualización. Esto como bien señala Luna (2005), implica integrar lo aprendido, reproducir los conocimientos, relacionarlos con otros y aplicarlos a la resolución de problemas de investigación. 
Asimismo desarrollamos la relación que el marco teórico tuvo con la elección de una metodología cualitativa centrada en análisis de documentos, observación y entrevistas estructuradas. Esas herramientas nos permitieron acceder a un corpus empírico que analizamos en torno a las categorías ligadas al concepto de identidad. Allí producimos un afinamiento de la teoría y construimos una serie de conceptos operacionales que nos permitieron pensar las dimensiones estática y dinámica, lo sedimentado y las reactivaciones de las identidades.

Pensar la dinámica política a partir de este marco teórico nos permitió analizar cómo los diversos grupos reestructuran sus identidades y cómo la constitución de límites y antagonismos surgidos en las distintas instancias posibilita el realineamiento y la rearticulación de los elementos discursivos en cada una de las configuraciones identitarias en busca de un nuevo intento de "sutura". Además nos brinda elementos para comprender que la dinámica política que comienza en 2003 no es de ruptura, cooptación y abandono del horizonte reivindicativo contestatario, sino que se trata de un proceso de construcción y reconfiguración de las identidades "nacional populares”.

El artículo presentó una serie de categorías teóricas y sus operacionalizaciones, que constituyen un esfuerzo de articulación entre una teoría de alto nivel de abstracción y el nivel empírico. En este sentido coincidimos con Sautú (2003) que debe haber un nexo entre el marco teórico, la formulación de objetivos y la metodología. Estas mediaciones entre lo abstracto y lo empírico, fueron conformando una batería de conceptos que nos permitieron pensar un concepto de identidad dinámico que recupere tanto las instancias sedimentadas como las rupturas y reconfiguraciones.

Este andamiaje teórico nos permitió observar que en distintos contextos es donde se vuelve a reconstruir la identidad a partir de la relectura del pasado y las expectativas que esa coyuntura despierta en las agrupaciones. En esos momentos decisorios las tradiciones y las identidades sedimentadas jugaron un rol central puesto que son estas las que les permitieron a las organizaciones significar las situaciones y escoger en consecuencia ciertas alternativas de acción a través de la decisión. Es central recuperar los sucesivos aprendizajes y posicionamientos, es decir, las experiencias políticas de las organizaciones para la explicación del proceso abierto en 2003, es decir rastrear su historicidad y sus imaginarios que los trasladan al plano de la disputa política. En este aspecto el análisis de la dinámica política a partir del concepto de identidad permite reconocer y explicar las acciones y a su vez deja abierta la agenda de investigaciones para distintos procesos históricos. 


\section{Notas}

${ }^{1}$ El presente trabajo constituye una relectura de las herramientas teóricas de la tesis de doctorado en Ciencias Sociales de FLACSO "La reconfiguración de las identidades "nacional populares" durante el kirchnerismo 2003-2009. Estudio de tres organizaciones: Libres del Sur, Movimiento Evita y Movimiento de Unidad Popular”, dirigida por el Dr. Aníbal Viguera y codirigida por el Dr. Martín Retamozo, a quienes se agradece las observaciones y comentarios.

${ }^{2}$ El campo “nacional popular” se constituye a partir de un conjunto de experiencias, imaginarios, figuras, mitos, símbolos y relatos ligados al peronismo, al nacionalismo revolucionario, al campo de las izquierdas y a miradas revisionistas de la historia, a los que las organizaciones apelarán de distinta forma configurando así sus identidades. Este "telón de fondo" es resignificado y atravesado por las identidades de las organizaciones que tomarán esos elementos articulando sus cadenas de significación.

${ }^{3}$ El movimiento "piquetero" es en realidad un movimiento de trabajadores desocupados de diversas tradiciones, ideologías y formatos organizativos (Svampa, 2004).

${ }^{4}$ El Partido Justicialista durante los años 90 implantó una serie de reformas neoliberales bajo la presidencia de Carlos Menem. Ese mismo partido impulsará una serie de reformas que darán lugar a un escenario posneoliberal.

${ }^{5}$ La etapa previa a los años 60` y 70’, se caracterizaba por la tendencia a la formación de grandes actores colectivos fundados e identificados con el mundo del trabajo. En el plano económico social, las ideologías intervencionistas y dirigistas, con algunos rasgos distintivos derivados de sus historias nacionales, fueron un elemento presente en la mayoría de las elites gubernamentales. El cierre de esta etapa mundial tuvo como principales procesos: en el Oeste, la crisis del Estado de Bienestar y la disminución de la importancia política y económica de la clase obrera industrial; en el Este, los colapsos de los socialismos reales; en el Sur las transiciones a la democracia (Sidicaro, 2003).

${ }^{6}$ La bibliografía sobre estas teorías es amplia y variada. Una síntesis bibliográfica de temas y autores puede encontrarse en Jenkins (1994).

${ }^{7}$ No obstante, es necesario aclarar que las perspectivas europeas y norteamericanas no representan "escuelas" teóricas unitarias, sino que defienden diferentes interpretaciones en sus explicaciones sobre el surgimiento y desarrollo de los movimientos sociales desde los setenta (Klandermans y Tarrow, 1988, citado en Rubio García, 2004).

${ }^{8}$ Según esta perspectiva, la sociedad es un mercado de recursos económicos, sociales e ideológicos y tanto los actores inconformes como sus adversarios desarrollan estrategias racionales para obtener la satisfacción de sus demandas o proteger sus intereses. De esta manera, el proceso central es el de la movilización de recursos que se conforma, por un lado, alrededor de individuos insatisfechos con un orden, que acumulan fuerza y desarrollan estrategias para incrementarla, y, por otro, por actores que defienden el orden y manejan el control social (Tarrés, 1992: 744).

${ }^{9}$ Cabe señalar que en los estudios sobre la acción colectiva popular en la Argentina reciente ha prevalecido el estudio de las identidades en los denominados movimientos sociales, con un énfasis en la identidad colectiva en su dimensión de identidad social en tanto represen- 
tantes de demandas de desocupados; el análisis de la identidad política de estas organizaciones ha recibido una atención menor.

${ }^{10}$ Brubaker y Cooper (2001) realizan tres críticas a lo que denominan la perspectiva constructivista. La primera se asienta en lo que llaman el constructivismo cliché. “Conceptos débiles de identidad son envueltos rutinariamente con calificativos estándar, indicando que la identidad es múltiple, inestable, en movimiento, contingente, fragmentada, construida, negociada, etc. Estos calificativos se han vuelto tan familiares -de hecho obligatoriosen los últimos años que uno los lee en forma automática”. La segunda crítica se basa en que no quedaría claro por qué los conceptos débiles de identidad son conceptos de identidad. La pregunta sería por qué utilizar un concepto que habla de igualdad y permanencia para dar cuenta de una realidad que es todo lo contrario. Por último, la tercera crítica se sustenta en que los conceptos débiles de identidad son demasiado débiles para ser empleados en trabajos teóricos. "En su preocupación por limpiar el término de sus connotaciones duras, en su insistencia de que las identidades son múltiples, maleables, fluidas, y así sucesivamente, los adeptos a la visión blanda de la identidad nos dejan con un término inútil para llevar a cabo un trabajo analítico serio”. (Brubaker y Cooper, 2001: 40)

11 Un estudio de caso implica un estudio profundizado y exhaustivo de uno o muy pocos objetos de investigación, lo que permite obtener un conocimiento amplio y detallado de los mismos. Resultan particularmente útiles cuando es necesario entender con detenimiento algún problema o situación específica, y cuando es posible identificar casos ricos y complejos en información. Según la tipología de Stake (1995) este trabajo puede identificarse como un estudio de caso instrumental, es decir que cumple el rol de mediación para la comprensión de un fenómeno que lo trasciende el caso en particular, es decir que los resultados pueden convertirse en un aporte para el análisis de alguna teoría o un fenómeno más general como lo es la participación ciudadana o el estudio de las relaciones entre el Estado y la sociedad. 


\section{Bibliografía}

Aboy Carlés, Gerardo (2001), Las dos fronteras de la democracia argentina. La reformulación de las identidades políticas de Alfonsín a Menem, Homo Sapiens, Rosario.

Alonso, Luis Enrique (1998), La mirada cualitativa en sociología, Editorial Fundamentos, Madrid.

Arfuch, Leonor (2002), “Problemáticas de la identidad”, en Arfuch, (Comp.) Identidades, sujetos y subjetividades, Prometeo, Buenos Aires.

Armelino, Martín (2007), Acción colectiva e historia. Notas para el estudio de la acción sindical de ATE (1976-2005). 4 Jornadas de Jóvenes Investigadores, organizadas por el Instituto de Investigaciones Gino Germani, Facultad de Ciencias Sociales (UBA), 19 al 21 de septiembre de 2007, Buenos Aires.

Barros, Sebastián (2006), "Espectralidad e inestabilidad institucional. Acerca de la ruptura populista”, en Estudios Sociales, año XVI, n ${ }^{\circ}$ 30, primer semestre, Santa Fé.

Bauman, Zigmunt (2002), Modernidad líquida, FCE, Buenos Aires.

Beck, Ulrich (2002), Libertad o capitalismo, Paidós, Barcelona.

Ídem (2004), Poder y contrapoder en la era global. La nueva economía política mundial, Paidós, Barcelona.

Biglieri, Paula y Perelló, Gloria (2007), En el nombre del Pueblo. La emergencia del populismo kirchnerista, Editorial de la Universidad de San Martín, Buenos Aires.

Brubaker, Rogers y Cooper, Frederick (2001), “Más allá de identidad”, en Apuntes de Investigación del CECYP. Año V, N 7, Fundación del Sur, Buenos Aires, pp. 30-67.

Canales, Francisca; Alvarado, Luz y Pineda, Elia (1989), Metodología para la investigación, Editorial Limusa.

Curzel, M. (2005), La complejidad de la revisión bibliográfica. En 2º Simposio electrónico Las Tres T (tesis, tesistas y tutores).

De La Garza, Enrique (1997), “Trabajo y Mundos de Vida”, en H. Zemelman (coord.) Subjetividad: Umbrales del Pensamiento Social, Anthropos, Madrid.

Ídem (2001), "La epistemología crítica y el concepto de configuración”, en Revista Mexicana de Sociología Nº1/2001, pp. 109-127. 
Dubar, Claude (1991), La socialización, Editorial Armand Colin, París.

Dubet, Francois (1989), "De la sociología de la identidad a la sociología del sujeto”, en Estudios Sociológicos, vol. VII, N²1, México D.F., pp. 519-545.

Giménez, Gilberto (1997), “Materiales para una teoría de las identidades sociales”. En Frontera Norte N ${ }^{0}$ 18. julio-diciembre, El Colegio de la Frontera Norte, México, pp. 9-28.

Ídem (2004), “Cultura e identidades”, en Revista Mexicana de Sociología, Año 66, $\mathrm{N}^{\circ}$ especial, México.

Grimson, Alejandro (2004), “La experiencia argentina y sus fantasmas”, en Grimson, Alejandro (comp.) La cultura en las crisis latinoamericanas, CLACSO, Buenos Aires, pp 177-193.

Gurrera, María Silvina (2005), “La redefinición del conflicto social. La conformación de la Central de Trabajadores Argentinos”, en Delamata Gabriela, Ciudadanía y Territorio. Las relaciones políticas de las nuevas identidades sociales, Editorial Espacio, Buenos Aires.

Jenkins, Craig (1994), “La teoría de la movilización de recursos y el estudio de los movimientos sociales”. En Zona Abierta, No 69, Madrid, pp. 548.

Klachko, Paula (2008), "Reflexiones generales sobre el movimiento de trabajadores desocupados”, en las Jornadas Internacionales de Problemas Latinoamericanos, Universidad Nacional de Mar del Plata.

Laclau, Ernesto (1990), Nuevas Reflexiones sobre la revolución de nuestro tiempo, Nueva Visión, Buenos Aires.

Ídem (2005), La razón populista, FCE, Buenos Aires.

Ídem (2006), "Por qué construir un pueblo es la tarea principal de la política radical”, en Cuadernos del CENDES, mayo-agosto año/vol. 23, núm., 062, políticas públicas, pp. 1-36. http://www.cendes-ucv.edu.ve/pdfs/revista62/cap1.pdf

Luna, H. (2005), “El escenario de la tesis”. En $2^{\circ}$ Simposio electrónico Las Tres T (tesis, tesistas y tutores). http://www.sai.com.ar/metodologia/ metodologia_simposio2.html

Mc Adam, Doug, Mc Carty, John, y Zald, Mayer (1999), “Oportunidades, estructuras de movilización y procesos enmarcadores: hacia una perspectiva sintética y comparada de los movimientos sociales”, en Movimientos sociales: perspectivas comparadas, Istmo, España. 
Melucci, Alberto (1994), “Asumir un compromiso: identidad y movilización en los movimientos sociales” en Zona-Abierta $N^{\circ}$ 69, Madrid.

Merklen, Denis (2005), Pobres ciudadanos: las clases populares en la era democrática 1983-2003, Gorla, Buenos Aires.

Munck, Gerardo (1995), “Algunos problemas conceptuales en el estudio de los movimientos sociales”, en Revista Mexicana de Sociología N³, pp. 17-39.

Muñoz, María Antonia (2006), "Laclau y Rancière: algunas coordenadas para la lectura de lo político”, Andamios. Revista de Investigación social. vol.2, N 4, México, pp.119-144.

Offe, Claus (1992), Partidos políticos y nuevos movimientos sociales, Sistema, Madrid.

Perelli, Carina (1986), Matrices ideológicas y discurso político: el problema de la hegemonía. Montevideo, CIESU, Documentos de Trabajo, n.125, Montevideo.

Pérez Ledesma, Manuel (1994), “Cuando lleguen los días de cólera (Movimientos sociales, teoría e historia)” en Zona-Abierta $N^{\circ}$ 69, Madrid.

Pizorno, Alessandro (1994), “Identidad e Interés”, en Zona Abierta, 69, Madrid, pp. 136-143.

Retamozo, Martín (2006), El movimiento de trabajadores desocupados en Argentina. Subjetividad y acción en la disputa por el orden social. Mimeo. Tesis de Doctorado. FLACSO, México.

Ídem (2008), "Los sentidos del (sin) trabajo. Subjetividad, demanda y acción colectiva de trabajadores desocupados en Argentina”, en Sociohistórica. Cuadernos del CISH, EDULP, La Plata, pp.55-89.

Ídem (2009a), Orden social, subjetividad y acción colectiva. Notas para el estudio de los movimientos sociales, en Athenea Digital: revista de pensamiento e investigación social, $\mathrm{N}^{\circ}$ 16, 2009, pags. 95-123.

Ídem (2009b), "Las Demandas Sociales y el Estudio de los Movimientos Sociales”, en Cinta de Moebio: Revista Electrónica de Epistemología de Ciencias Sociales, $\mathrm{N}^{\mathrm{o}} 35$.

Sautú, Ruth (2003), Todo es teoría. Objetivos y métodos de investigación. Buenos Aires: Editorial Lumiere.

Schuster, Federico (2005), “Las protestas sociales y el estudio de la acción colectiva”, en Schuster y otros (comps.) Tomar la palabra. Estudios sobre protesta social y acción colectiva en la Argentina contemporánea, Prometeo, Buenos Aires. 
Sidicaro, Ricardo (2003), “Consideraciones sociológicas sobre la Argentina en la Segunda Modernidad”, en Estudios sociales, N.24, primer semestre.

Stake, R. E. (1995), Investigación con estudio de casos. Morata, Madrid.

Svampa, Maristella (2000) “Identidades astilladas. De la patria metalúrgica al heavy metal” en Svampa, M. (ed.) Desde abajo. Las transformaciones de las identidades sociales, Biblos-UNGS, Buenos Aires, pp. 121 154.

Ídem (2004), “Cinco Tesis sobre la nueva matriz popular”, en revista Lavboratorio/n line. Revista de Estudios Sobre Cambio Social año IV. número15. primavera.

Ídem (2005), “La Sociedad Excluyente. La Argentina bajo el signo del neoliberalismo”, Taurus, Buenos Aires.

Tarrés, María Luisa (1992), “Perspectivas analíticas en la sociología de la acción colectiva”, en Estudios Sociológicos, Volumen 10, № 30, Septiembre-diciembre.

Tarrow, Sydney (1997), El poder en movimiento, Alianza, Madrid.

Ídem (1999) “Estado y oportunidades: la estructuración política de los movimientos sociales” en Mc Adam, Doug, Mc Carty, John, y Zald, Mayer, Movimientos sociales: perspectivas comparadas, Istmo, España.

Touraine, Alain (1991), Los Movimientos Sociales, Editorial Almagesto, México.

Torres Carrillo, Alfonso (2006), “Organizaciones populares, construcción de identidad y acción política”, en Revista Latinoamericana de Ciencias Sociales, Vol $4 \mathrm{~N}^{\mathrm{o}}$ 2, Universidad de Manizales, Colombia.

Zemelman, Hugo (1995), "La esperanza como conciencia (un alegato contra el bloqueo histórico imperante: ideas sobre sujetos y lenguaje)”, en Zemelman (coord.) Determinismos y Alternativas en las Ciencias Sociales de América Latina, CRIM-UNAM, México.

Ídem (1997), “Sujetos y subjetividad en la construcción metodológica”, en León y Zemelman (coords.), Subjetividad: Umbrales del Pensamiento Social, Anthropos, Barcelona, pp.21-35. 